Research Article

\title{
A Study on a New Class of Backward Stochastic Differential Equation
}

\author{
Ping He $\left.\mathbb{D}^{1}\right)^{1,2}$ Yong Ren ${ }^{(D)},{ }^{1}$ and Defei Zhang $\mathbb{D}^{2}$ \\ ${ }^{1}$ Department of Mathematics, Anhui Normal University, Wuhu 241000, China \\ ${ }^{2}$ Department of Mathematics, Honghe University, Mengzi 661199, China
}

Correspondence should be addressed to Defei Zhang; zhdefei@163.com

Received 10 January 2020; Accepted 7 April 2020; Published 21 May 2020

Academic Editor: Mariusz Michta

Copyright $(2020$ Ping He et al. This is an open access article distributed under the Creative Commons Attribution License, which permits unrestricted use, distribution, and reproduction in any medium, provided the original work is properly cited.

The existence and uniqueness for a new type of backward stochastic differential equation when the generator includes the values of solutions of the past, the present, and the future are obtained in this paper. An important comparison theorem for this sort of BSDEs is also proved.

\section{Introduction}

Pardoux and Peng in [1] first provided the famous backward stochastic differential equations (BSDEs). The existence and uniqueness for the BSDEs are proved by them. Since then, BSDEs have been discussed and applied to many fields, e.g., Chen and Epstein [2] and Karoui et al. [3-7]. A lot of research has focused on the assumptions on the generator, such as [8-12]. Recently, Delong and Imkeller in $[13,14]$ obtained many interesting results about the time-delayed equation in which the generator at time $t$ only depends on the past solution. Peng and Yang in [15] discussed anticipated BSDEs, in which the generator includes present and future solutions.

Therefore, the natural questions are as follows: can we discuss the backward stochastic differential equations when the generator includes not only the past and the present but also the future solutions? The comparison theorem for it is still true? Indeed, these questions are answered in the affirmative in this paper. The equation is called delay and anticipated BSDEs (DABSDEs for short). We obtain existence and uniqueness for delay and anticipated BSDEs, which can be seen as a general version of Delong and Imkeller in [13] or Peng and Yang in [15].

\section{Main Notations}

Let $(\Omega, \mathscr{F}, P)$ be a probability space equipped with a natural filtration $\left(\mathscr{F}_{v}\right)_{v \geq 0}$ and $\left(B_{v}\right)_{v \geq 0}$ be a standard Brownian motion. We denote the norm in $R^{m}$ by $|\cdot|$. Given $T>0$, denote the following:

(i) $L^{2}\left(\mathscr{F}_{T} ; R^{n}\right)=\left\{\xi: \mathscr{F}_{T}\right.$-measurable and $\left.E\left[|\xi|^{2}\right]<\infty\right\}$.

(ii) $L_{\mathscr{F}}^{2}\left(0, T ; R^{n}\right)=\left\{\varphi_{t}: \mathscr{F}_{t}\right.$-adapted and $E\left[\int_{0}^{T}\left|\varphi_{t}\right|^{2} \mathrm{~d} t\right]$ $<\infty\}$.

(iii) $S_{\mathscr{F}}^{2}\left(0, T ; R^{n}\right)=\left\{\chi_{s}: \chi_{s} \in L_{\mathscr{F}}^{2}\left(0, T ; R^{n}\right)\right.$ and continuous, as well as $\left.E\left[\sup \left|\chi_{t}\right|^{2}\right]<\infty\right\}$.

In the case $n=1$, they are abbreviated to $L^{2}\left(\mathscr{F}_{T}\right), L_{\mathscr{F}}^{2}(0, T)$, and $S_{\mathscr{F}}^{2}(0, T)$, respectively.

\section{Delay and Anticipated Backward Stochastic Differential Equations}

We propose a new type of BSDEs as follows: 


$$
\begin{cases}-d Y_{t}=g\left(t, Y_{t-d_{1}(t)}, Z_{t-d_{2}(t)}, Y_{t}, Z_{t}, Y_{t+d_{3}(t)}, Z_{t+d_{4}(t)}\right) \mathrm{d} t-Z_{t} d B_{t}, & 0 \leq t \leq T, \\ Y_{t}=\xi_{t}, & T \leq t \leq T+D, \\ Z_{t}=\eta_{t}, & T \leq t \leq T+D,\end{cases}
$$

where $d_{i}(\cdot), i=1, \ldots, 4$, are four continuous functions s.t.

$$
\begin{aligned}
& \text { (D1) } \exists D \geq 0 \text { s.t. } \\
& \begin{aligned}
0 \leq t & -d_{1}(t) \leq t ; 0 \leq t-d_{2}(t) \leq t ; t+d_{3}(t) \leq T+D ; t \\
& +d_{4}(t) \leq T+D, 0 \leq t \leq T .
\end{aligned}
\end{aligned}
$$

(D2) $\exists L \geq 0$ s.t., for all nonnegative and integrable $f(\cdot)$,

$$
\begin{aligned}
\int_{t}^{T} f\left(v-d_{1}(v)\right) \mathrm{d} v & \leq L \int_{t}^{T+D} f(v) \mathrm{d} v ; \int_{t}^{T} f\left(v-d_{2}(v)\right) \mathrm{d} v \\
& \leq L \int_{t}^{T+D} f(v) \mathrm{d} v \\
\int_{t}^{T} f\left(v+d_{3}(v)\right) \mathrm{d} v & \leq L \int_{t}^{T+D} f(v) \mathrm{d} v ; \int_{t}^{T} f\left(v+d_{4}(v)\right) \mathrm{d} v \\
& \leq L \int_{t}^{T+D} f(v) \mathrm{d} v .
\end{aligned}
$$

Note that the simple examples for $d_{i}(s)$ satisfying $(D 1)$ and $(D 2)$ are constant delay and $d_{i}(s)=s$.

We call system (1) delay and anticipated backward stochastic differential equations (delay and anticipated BSDEs).

Our aim is to search out a pair of processes $(Y ., Z.) \in S_{\mathscr{F}}^{2}\left(0, D+T ; R^{m}\right) \times L_{\mathscr{F}}^{2}\left(0, D+T ; R^{m \times d}\right) \quad$ which satisfies the delay and anticipated BSDEs (1).

For all $s \in[0, T]$, we suppose $g(\omega, s, \phi, \mu, y, z, \psi, v): \Omega \times$ $[0, T] \times L_{\mathscr{F}}^{2}\left(0, s ; \quad R^{m}\right) \times L_{\mathscr{F}}^{2}\left(0, s ; R^{m \times d}\right) \times R^{m} \times R^{m \times d} \times L^{2}$
$\left(\mathscr{F}_{r} ; R^{m}\right) \times L^{2} \quad\left(\mathscr{F}_{r^{\prime}} ; R^{m \times d}\right) \longrightarrow L^{2}\left(\mathscr{F}_{s} ; R^{m}\right), \quad s \leq r, r^{\prime} \leq T+$ $D$, and satisfies

(A1) $\exists C>0, \quad \phi, \phi^{\prime} \in L_{\mathscr{F}}^{2}\left(0, s ; R^{m}\right) ; \mu, \mu^{\prime} \quad \in L_{\mathscr{F}}^{2}(0, s ;$ $\left.R^{m \times d}\right) ; y, y^{\prime} \in R^{m} ; z, z^{\prime} \in R^{m \times d} ; \quad \psi, \psi^{j} \in L_{\mathscr{F}}^{2}(s, T+D ;$ $\left.R^{m}\right) ; \nu, v^{\prime} \in \quad L_{\mathscr{F}}^{2}\left(s, T+D ; R^{m \times d}\right) ; r, t \in[0, s] ; \bar{r}, \bar{t} \in$ $[s, T+D], 0 \leq s \leq T$, such that

$$
\begin{aligned}
& \left|g\left(s, \phi_{r}, \mu_{t}, y, z, \psi_{\bar{r}}, \nu_{\bar{t}}\right)-g\left(s, \phi_{r}^{\prime}, \mu_{t}^{\prime}, y^{\prime}, z^{\prime}, \psi_{\bar{r}}^{\prime}, v_{\bar{t}}^{\prime}\right)\right| \\
& \leq C\left(\left|\phi_{r}-\phi_{r}^{\prime}\right|+\left|\mu_{t}-\mu_{t}^{\prime}\right|+\left|y-y^{\prime}\right|+\left|z-z^{\prime}\right|\right. \\
& \left.\quad+E\left[\left|\psi_{\bar{r}}-\psi_{\bar{r}}^{\prime}\right|+\left|\nu_{\bar{t}}-v_{\bar{t}}^{\prime}\right| \mid \mathscr{F}_{s}\right]\right) . \\
& \text { (A2) } E\left[\int_{0}^{T}|g(s, 0,0,0,0,0,0)|^{2}\right]<\infty .
\end{aligned}
$$

Theorem 1. Suppose $g$ satisfies (A1) and (A2), as well as $d_{i}(t), i=1, \ldots, 4$, satisfy $(D 1)$ and (D2). Then, the DABSDE (1) has a unique solution for $\xi \in S_{\mathscr{F}}^{2}\left(T, D+T ; R^{m}\right)$ and $\eta \in L_{\mathscr{F}}^{2}\left(T, D+T ; R^{m \times d}\right)$, namely, there exists unique $(\widetilde{Y}, \widetilde{Z}) \in S_{\mathscr{F}}^{2}\left(0, D+T ; R^{m}\right) \times L_{\mathscr{F}}^{2}\left(0, D+T ; R^{m \times d}\right)$ satisfying the DABSDEs.

Proof. We choose suitable $\beta$ which satisfies $0<\left(e^{\beta T} / \beta\right) \leq 1 /\left(32 C^{2}(2 L+1)\right)$ and define a norm in $L_{\mathscr{F}}^{2}\left(0, D+T ; R^{m}\right)$ :

$$
\|\mu(\cdot)\|_{\beta}=\sqrt{E\left[\int_{0}^{D+T}\left|\mu_{t}\right|^{2} e^{\beta t} \mathrm{~d} t\right]} .
$$

We set

$$
\begin{cases}Y_{u}=\xi_{T}+\int_{u}^{T} g\left(v, y_{v-d_{1}(v)}, z_{v-d_{2}(v)}, y_{v}, z_{v}, y_{v+d_{3}(v)}, z_{v+d_{4}(v)}\right) \mathrm{d} v-\int_{u}^{T} Z_{v} \mathrm{~d} B_{v}, & 0 \leq u \leq T \\ Y_{u}=\xi_{u}, & T \leq u \leq T+D \\ Z_{u}=\eta_{u}, & T \leq u \leq T+D .\end{cases}
$$

We set a mapping $M[(y, z)]:=(Y, Z): L_{\mathscr{F}}^{2}$ $\left(0, D+T ; R^{m} \times R^{m \times d}\right) \longrightarrow L_{\mathscr{F}}^{2}\left(0, D+T ; R^{m} \times R^{m \times d}\right)$. Let us show $M$ is a contraction mapping under $\|\cdot\|_{\beta}$. $\forall(y, z),\left(y_{\prime \prime}, z_{\prime \prime}\right) \in L_{\mathscr{F}}^{2}\left(0, T+D ; R^{m} \times R^{m \times d}\right) ; \quad$ define $(Y, Z)=M\left(y^{\prime}, z\right),\left(Y^{\prime}, Z^{\prime}\right)=M\left(y^{\prime}, z^{\prime}\right)$, and

$$
\begin{aligned}
\left(\widehat{y}_{.}, \widehat{z}_{.}\right) & =\left(y-y_{.}^{\prime}, z-z_{.}^{\prime}\right) \\
\left(\widehat{Y}_{.}, \widehat{Z} .\right) & =\left(Y-Y_{.}^{\prime}, Z-Z_{.}^{\prime}\right)
\end{aligned}
$$

Using Itô's lemma for $\left|\widehat{Y}_{u}\right|^{2} e^{\beta u}, 0 \leq u \leq T$, and taking expectation, 


$$
\begin{aligned}
\left|\widehat{Y}_{0}\right|^{2}+\beta E\left[\int_{0}^{T} e^{\beta u}\left|\widehat{Y}_{u}\right|^{2} \mathrm{~d} s\right]+E\left[\int_{0}^{T} e^{\beta u}\left|\widehat{Z}_{u}\right|^{2} \mathrm{~d} u\right] \\
=E\left[2 \int _ { 0 } ^ { T } e ^ { \beta u } \left(g\left(u, y_{u-d_{1}(u)}, z_{u-d_{2}(u)}, y_{u}, z_{u}, y_{u+d_{3}(u)}, z_{u+d_{4}(u)}\right)\right.\right. \\
\left.\left.\quad-g\left(u, y_{u-d_{1}\left(u^{\prime}\right)}, z_{s-d_{2}\left(u^{\prime}\right)}, y_{u}^{\prime}, z_{u^{\prime}}^{\prime}, y_{u+d_{3}\left(u^{\prime}\right)}, z_{u+d_{4}\left(u^{\prime}\right)}\right)\right) \widehat{Y}_{u} \mathrm{~d} u\right] \\
\leq \frac{\beta}{2} E\left[\int_{0}^{T} e^{\beta u}\left|\widehat{Y}_{u}\right|^{2} \mathrm{~d} u\right]+\frac{2}{\beta} E\left[\int_{0}^{T} e^{\beta u} \mid g\left(u, y_{u-d_{1}(u)}, z_{u-d_{2}(u)}, y_{u}, z_{u}, y_{u+d_{3}(u)}, z_{u+d_{4}(u)}\right)\right. \\
\quad-g\left(u, y_{u-d_{1}(u)^{\prime}}, z_{u-d_{2}\left(u^{\prime}\right)}, y_{u}^{\prime}, z_{u^{\prime}}^{\prime}, y_{u+d_{3}\left(u^{\prime}\right)},\left.z_{\left.u+d_{4}(u)^{\prime}\right)}\right|^{2} \mathrm{~d} u\right] .
\end{aligned}
$$

Thus,

$$
\begin{aligned}
& E\left[\int_{0}^{T} e^{\beta u}\left(\frac{\beta}{2}\left|\widehat{Y}_{u}\right|^{2}+\left|\widehat{Z}_{u}\right|^{2} \mathrm{~d} u\right]\right. \\
& \leq \frac{2}{\beta} E\left[\int_{0}^{T} e^{\beta u} \mid g\left(u, y_{u-d_{1}(u)}, z_{u-d_{2}(u)}, y_{u}, z_{u}, y_{u+d_{3}(u)}, z_{u+d_{4}(u)}\right)\right. \\
& \left.\quad-\left.g\left(u, y_{u-d_{1}\left(u^{\prime},\right.}, z_{u-d_{2}(u)^{\prime}}, y_{u}^{\prime}, z_{u}^{\prime}, y_{u+d_{3}\left(u^{\prime}\right)}, z_{u+d_{4}\left(u^{\prime}\right)}\right)\right|^{2} \mathrm{~d} u\right] .
\end{aligned}
$$

\section{First, we note that}

$$
\begin{aligned}
\int_{0}^{T} e^{\beta s}\left|\widehat{y}_{s-d_{i}(s)}\right|^{2} \mathrm{~d} s & =\int_{0}^{T} e^{\beta\left(s-d_{i}(s)\right)}\left|\widehat{y}_{s-d_{i}(s)}\right|^{2} e^{\beta d_{i}(s)} \mathrm{d} s \\
& \leq e^{\beta T} \int_{0}^{T} e^{\beta s}\left|\widehat{y}_{s}\right|^{2} \mathrm{~d} s .
\end{aligned}
$$

Because $d_{i}(s), i=1, \ldots, 4$, satisfy (D2) and $g$ satisfies $(A 1)$, we have

$$
\begin{aligned}
& E\left[\int_{0}^{T} e^{\beta s}\left(\frac{\beta}{2}\left|\widehat{Y}_{s}\right|^{2}+|\widehat{Z}|^{2} \mathrm{~d} s\right]\right. \\
& \quad \leq \frac{2 C^{2}}{\beta} E\left[\int_{0}^{T} e^{\beta s}\left(\left|\widehat{y}_{s-d_{1}(s)}\right|+\left|\widehat{z}_{s-d_{2}(s)}\right|+\left|\widehat{y}_{s}\right|+\left|\widehat{z}_{s}\right|+E\left[\left|\widehat{y}_{s+d_{3}(s)}\right|+\left|\widehat{z}_{s+d_{4}(s)}\right| \mid \mathscr{F}_{s}\right]\right)^{2} \mathrm{~d} s\right] \\
& \quad \leq \frac{8 C^{2}}{\beta} E\left[\int_{0}^{T} e^{\beta u}\left(2\left|\widehat{y}_{u-d_{1}(u)}\right|^{2}+2\left|\widehat{z}_{u-d_{2}(u)}\right|^{2}+\left|\widehat{z}_{u}\right|^{2}+\left|\widehat{y}_{u}\right|^{2}+2\left|\widehat{y}_{u+d_{3}(u)}\right|^{2}+2\left|\widehat{z}_{u+d_{4}(u)}\right|^{2}\right) \mathrm{d} u\right] \\
& \quad \leq \frac{8 C^{2}(2 L+1) e^{\beta T}}{\beta} E\left[\int_{0}^{T+D} e^{\beta u}\left(\left|\widehat{y}_{u}\right|^{2}+\left|\widehat{z}_{u}\right|^{2}\right) \mathrm{d} u\right] .
\end{aligned}
$$

Since $\beta$ satisfies $\left(e^{\beta T} / \beta\right) \leq 1 /\left(32 C^{2}(2 L+1)\right)$, then

$$
\begin{aligned}
& E\left[\int_{0}^{T+D} e^{\beta s}\left(\left|\widehat{Y}_{s}\right|^{2}+|\widehat{Z}|^{2}\right) \mathrm{d} s\right] \leq E\left[\int_{0}^{T+D} e^{\beta s}\left(\frac{\beta}{2}\left|\widehat{Y}_{s}\right|^{2}+|\widehat{Z}|^{2} \mathrm{~d} s\right]\right. \\
& \quad \leq \frac{1}{4} E\left[\int_{0}^{T+D} e^{\beta s}\left(\left|\hat{y}_{s}\right|^{2}+\left|\widehat{z}_{s}\right|^{2}\right) \mathrm{d} s\right] .
\end{aligned}
$$

Therefore,

$$
\|(\widehat{Y}, \widehat{Z} .)\|_{\beta} \leq \frac{1}{2}\|(\widehat{y}, \widehat{z} .)\|_{\beta} .
$$

Consequently, $M$ is a strict contraction mapping. From the fixed point theorem, the DABSDE (1) has a unique solution.
We use Itô's lemma for $\left|Y_{v}\right|^{2} e^{\alpha v}, t \leq v \leq T$, where $\alpha$ is an arbitrary constant, and take conditional expectation, similar to Proposition 4.4 in [15]; then,

$$
\begin{aligned}
& E\left[\sup _{0 \leq t \leq T}\left|Y_{t}\right|^{2}+\int_{t}^{T}\left|Z_{s}\right|^{2} \mathrm{~d} s \mid \mathscr{F}_{t}\right] \leq C^{\prime} E\left[\left|\xi_{T}\right|^{2}\right. \\
& \left.\quad+\int_{0}^{T}|g(s, 0,0,0,0,0,0)|^{2} \mathrm{~d} s+\int_{T}^{T+D}\left(\left|\xi_{v}\right|^{2}+\left|\eta_{v}\right|^{2}\right) \mathrm{d} v\right]
\end{aligned}
$$

where $C^{\prime}$ only depends on $C$ in $(A 1), L$ in (D2), and $T$. Therefore, $Y \in S_{\mathscr{F}}^{2}\left(0, D+T ; R^{m}\right)$.

Example 1. Consider a typical delay and anticipated backward stochastic differential equation 


$$
\begin{cases}-d Y_{u}=\left(Z_{u-(\pi / 2)}+Z_{u+\pi}\right) \mathrm{d} u-Z_{u} \mathrm{~d} B_{u}, & 0 \leq u \leq T, \\ Y_{u}=\int_{0}^{T} \sin t \mathrm{~d} B_{t}, & T \leq u \leq T+D, \\ Z_{u}=\sin T, & T \leq u \leq T+D,\end{cases}
$$

with $Y_{T}=\int_{0}^{T} \sin t \mathrm{~d} B_{t}$. We can get the unique solution of DABSDE (15) which is

$$
\begin{cases}Y_{u}=\int_{0}^{u} \sin s \mathrm{~d} B_{s}-(\cos u+\sin u)+\cos T+\sin T, & 0 \leq u \leq T, \\ Z_{u}=\sin u, & 0 \leq u \leq T .\end{cases}
$$

\section{Comparison Theorem for Delay and Anticipated BSDEs}

Next, we deduce the comparison theorem for one-dimensional DABSDEs. Denote $(i)_{t}^{(i)}:=Y_{t}^{(i)}, i=1,2,3$. Let $\left(Y^{(j)}, Z^{(j)}\right)(j=1,2)$ be the solution of the following onedimensional delay and anticipated BSDEs:

$$
\begin{cases}-d Y_{s}^{(j)}=g_{j}\left(s, Y_{s-d_{1}(s)}^{(j)}, Y_{s}^{(j)}, Z_{s}^{(j)}, Y_{s+d_{3}(s)}^{(j)}\right) \mathrm{d} s-Z_{s}^{(j)} d B_{s}, & s \in[0, T] \\ Y_{s}^{(j)}=\xi_{s}^{(j)}, & s \in[T, D+T]\end{cases}
$$

$$
Y_{t}^{(2)} \leq Y_{t}^{(1)}, \quad \text { a.e., a.s. }
$$

Theorem 2. Assume that $g_{1}, g_{2}$ satisfy (A1) and (A2), $\xi^{(1)}, \xi^{(2)} \in S_{\mathscr{F}}^{2}(T, D+T), d_{k}(t), k=1,3$, satisfy (D1), (D2), and $\forall y \in R, z \in R^{d}, g_{2}(t, \cdot, y, z, \cdot)$ is strictly increasing. If $\xi^{(2)} \leq \xi^{(1)}, s \in[T, D+T] \quad$ and $\quad g_{1}\left(\cdot, \phi_{r}, y, z, \varphi_{\bar{r}}\right) \geq g_{2}$ $\left(\cdot, \phi_{r}, y, z, \varphi_{\bar{r}}\right) \quad, \phi_{r} \in L_{\mathscr{F}}^{2}(0, t), \varphi_{\bar{r}} \in L_{\mathscr{F}}^{2}(t, T+D), r \in$ $[0, t], \bar{r} \in[t, D+T]$, then
In particular, we also have the strict comparison theorem:

$$
Y_{0}^{(1)}=Y_{0}^{(2)} \Longleftrightarrow \begin{cases}g_{1}\left(t, Y_{t-d_{1}(t)}^{(1)}, Y_{\cdot}^{(1)}, Z_{\cdot}^{(1)}, Y_{t+d_{3}(t)}^{(1)}\right)=g_{2}\left(t, Y_{t-d_{1}(t)}^{(1)}, Y_{\cdot}^{(1)}, Z_{\cdot}^{(1)}, Y_{t+d_{3}(t)}^{(1)}\right), & t \in[0, T] \\ \xi_{t}^{(1)}=\xi_{t}^{(2)}, & t \in[T, D+T]\end{cases}
$$

Proof. Set

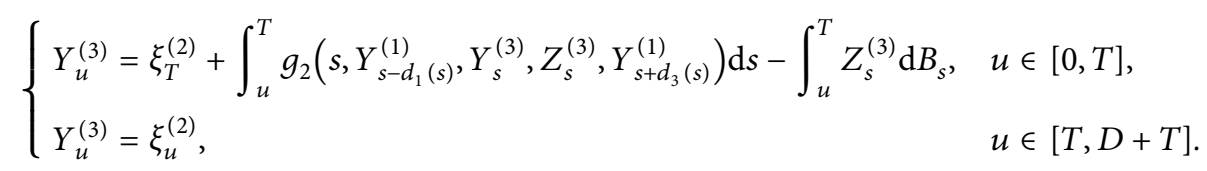

Denote $\widehat{g}_{u}:=g_{1}\left(u, Y_{u-d_{1}(u)}^{(1)}, Y_{u}^{(1)}, Z_{u}^{(1)}, Y_{u+d_{(2)}}^{(1)}\right)-g_{2}$
$\left(u, Y_{u-d_{(1)}}^{(1)}, Y_{u}^{(1)}, Z_{u}^{(1)}, Y_{u+d_{3}(u)}^{(1)}\right)$ and $\xi^{(1)} \stackrel{\xi^{(2)}}{=}, \widehat{y}:=$ $Y^{(1)}-Y^{(3)}, \widehat{z}:=Z^{(1)}-Z^{(3)}$. Then, $(\hat{y}, \widehat{z})$ is the solution of the following DABSDEs:

$$
\begin{cases}\widehat{y}_{t}=\widehat{\xi}_{T}+\int_{t}^{T}\left(\widehat{g}_{v}+a_{v} \widehat{y}_{v}+b_{v} \widehat{z}_{v}\right) \mathrm{d} v-\int_{t}^{T} \widehat{z}_{v} \mathrm{~d} B_{v}, & 0 \leq t \leq T, \\ y_{t}=\widehat{\xi}_{t}, & T \leq t \leq D+T,\end{cases}
$$


where

$$
\begin{aligned}
& a_{v}= \begin{cases}\frac{g_{1}\left(v, Y_{v-d_{1}(v)}^{(1)}, Y_{v}^{(1)}, Z_{v}^{(1)}, Y_{v+d_{3}(v)}^{(1)}\right)-g_{2}\left(v, Y_{v-d_{1}(v)}^{(1)}, Y_{v}^{(3)}, Z_{v}^{(1)}, Y_{v+d_{3}(v)}^{(1)}\right)}{Y_{v}^{(1)}-Y_{v}^{(3)}}, & Y_{v}^{(1)} \neq Y_{v}^{(3),} \\
0, & Y_{v}^{(1)}=Y_{v}^{(3)},\end{cases} \\
& b_{v}= \begin{cases}\frac{g_{1}\left(v, Y_{v-d_{1}(v)}^{(1)}, Y_{v}^{(3)}, Z_{v}^{(1)}, Y_{v+d_{3}(v)}^{(1)}\right)-g_{2}\left(v, Y_{v-d_{1}(v)}^{(1)}, Y_{v}^{(3)}, Z_{v}^{(3)}, Y_{v+d_{3}(v)}^{(1)}\right)}{Z_{v}^{(1)}-Z_{v}^{(3)},} & Z_{v}^{(1)} \neq Z_{v}^{(3)}, \\
0, & Z_{v}^{(1)}=Z_{v}^{(3)},\end{cases} \\
& \hat{y}_{t}=E\left[\widehat{\xi}_{T} q_{T}+\int_{t}^{T} \widehat{g}_{s} q_{s} \mathrm{~d} s \mid \mathscr{F}_{t}\right] .
\end{aligned}
$$

Since $g_{2}$ follows assumption $(A 1)$, then $\left|a_{\nu}\right| \leq C$ and $\left|b_{v}\right| \leq C$. Denote

$$
q_{s}:=\exp \left[-\frac{1}{2} \int_{0}^{s}\left|b_{v}\right|^{2} \mathrm{~d} v+\int_{0}^{s} a_{v} \mathrm{~d} v+\int_{0}^{s} b_{v} \mathrm{~d} B_{v}\right] .
$$

Using Itô's lemma for $q_{s} \hat{y}_{s}$ and taking expectation, then
From this, we have $Y^{(3)} \leq Y^{(1)}$ a.e., a.s.

Similarly, we set

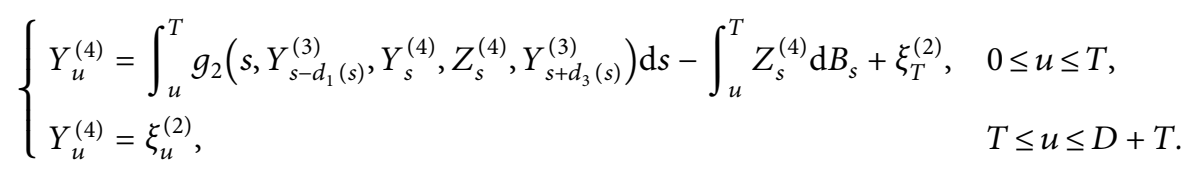

Because $Y_{t}^{(1)} \geq Y_{t}^{(3)}$ a.e., a.s., and $g_{2}(\cdot)$ is strictly increasing, from the classical comparison theorem, then $Y^{(4)} \leq Y^{(3)}$. When $n=5,6, \ldots$, we investigate the DABSDEs:

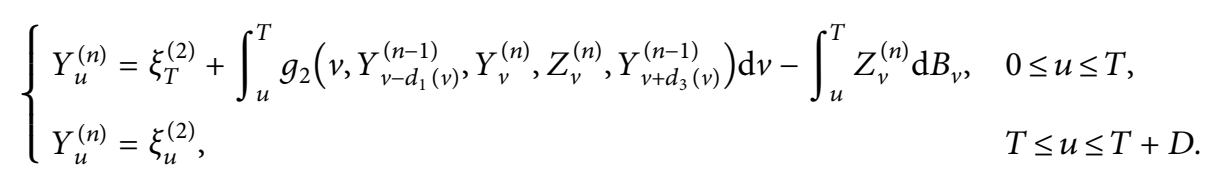

Since $g_{2}(t, \cdot, y, z, \cdot)$ is strictly increasing, we have $Y_{t}^{(4)} \geq Y_{t}^{(5)} \geq \cdots \geq Y_{t}^{(5)} \geq$. From the proving method of Theorem 1, $\left(Y^{(n)}\right)$ and $\left(Z^{(n)}\right)_{n \geq 4}$ are Cauchy sequences in
$L_{\mathscr{F}}^{2}(0, D+T)$ and in $L_{\mathscr{F}}^{2}(0, T), n \geq 4$. Write their limits as $Y$. and $Z$; then, $(Y, Z) \in L_{\mathscr{F}}^{2}(0, D+T) \times L_{\mathscr{F}}^{2}(0, T)$, and when $n \longrightarrow \infty$,

$$
\begin{gathered}
E\left[\int_{t}^{T}\left|g_{2}\left(s, Y_{s-d_{1}(s)}^{(n-1)}, Y_{s}^{(n)}, Z_{s}^{(n)}, Y_{s+d_{3}(s)}^{(n-1)}\right)-g_{2}\left(s, Y_{s-d_{1}(s)}, Y_{s}, Z_{s}, Y_{s+d_{3}(s)}\right)\right|^{2} e^{\beta s} \mathrm{~d} s\right] \\
\quad \leq 4 C^{2} E\left[\int_{t}^{T}\left(\left|Y_{v}^{(n)}-Y_{v}\right|^{2}+2 L\left|Y_{v}^{(n-1)}-Y_{v}\right|^{2}+\left|Z_{v}^{(n)}-Z_{v}\right|^{2}\right) e^{\beta v}\right] \longrightarrow 0 .
\end{gathered}
$$


Therefore, $(Y, Z)$ satisfies the following delay and anticipated BSDEs:

$$
\begin{cases}Y_{t}=\xi_{T}^{(2)}+\int_{t}^{T} g_{2}\left(s, Y_{s-d_{1}(s)}, Y_{s}, Z_{s}, Y_{s+d_{3}(s)}\right) \mathrm{d} s-\int_{t}^{T} Z_{s} \mathrm{~d} B_{s}, & 0 \leq t \leq T \\ Y_{t}^{(n)}=\xi_{t}^{(2)}, & T \leq t \leq T+D .\end{cases}
$$

By Theorem 1, $Y .=Y^{(2)}$.

Since $Y_{t}^{(1)} \geq Y_{t}^{(3)} \geq \cdots \geq Y_{t}^{(n)} \geq \cdots$, it holds immediately $Y^{(1)} \geq Y^{(2)}$.

Similar to the deducing technique of Peng and Yang [15], next we prove the strict comparison theorem.

If $Y_{0}^{(1)}=Y_{0}^{(2)}$, then we have

$$
\begin{aligned}
& g_{1}\left(u, Y_{u-d_{1}(u)}^{(1)}, Y_{u}^{(1)}, Z_{u}^{(1)}, Y_{u+d_{3}(u)}^{(1)}\right) \\
& \quad=g_{2}\left(u, Y_{u-d_{1}(u)}^{(2)}, Y_{u}^{(1)}, Z_{u}^{(1)}, Y_{u+d_{3}(u)}^{(2)}\right),
\end{aligned}
$$

Since $Y_{0}^{(1)} \geq Y_{0}^{(3)} \geq Y_{0}^{(2)}$, then $Y_{0}^{(1)}=Y_{0}^{(3)}$ and

$$
\begin{aligned}
& g_{1}\left(t, Y_{t-d_{1}(t)}^{(1)}, Y_{t}^{(1)}, Z_{t}^{(1)}, Y_{t+d_{3}(t)}^{(1)}\right) \\
& \quad=g_{2}\left(t, Y_{t-d_{1}(t)}^{(1)}, Y_{t}^{(1)}, Z_{t}^{(1)}, Y_{t+d_{3}(t)}^{(1)}\right),
\end{aligned}
$$

as well as $g_{2}(\cdot)$ is strictly increasing, and we can easily get satisfying results.
If $\quad g_{1}\left(u, Y_{u-d_{1}(u)}^{(1)}, Y_{u}^{(1)}, Z_{u}, Y_{u+d_{3}(u)}^{(1)}\right)=g_{2}(u, Y \wedge$ $\left.(1)_{u-d_{1}(u)}^{(1)}, Y_{u}^{(1)}, Z_{u}^{(1)}, Y_{u+d_{3}(u)}^{(1)}\right), 0 \leq u \leq T$, and $\xi_{u}^{(1)}=\xi_{u}^{(2)}$, $T \leq u \leq T+D$, then

$$
\widehat{y}_{t}=Y_{t}^{(1)}-Y_{t}^{(3)}=E\left[\widehat{\xi}_{T} q_{T}+\int_{t}^{T} \widehat{g}_{s} q_{s} \mathrm{~d} s \mid \mathscr{F}_{t}\right] \equiv 0 .
$$

Therefore, from Theorem $1, Y^{(1)}=Y^{(2)}$; in particular, $Y_{0}^{(1)}=Y_{0}^{(2)}$, a.e., a.s.

The following result is to show the important relation between the delayed time and the solution of delay and anticipated BSDEs.

Theorem 3. Suppose $\left(Y^{(l)}, Z^{(l)}\right), l=1,2$, are solutions of two delay and anticipated BSDEs:

$$
\begin{cases}-d Y_{s}^{(l)}=g\left(s, Y_{s-d_{1}^{(l)}(s)}^{(l)}, Y_{s}^{(l)}, Z_{s}^{(l)}, Y_{s+d_{3}^{(l)}(s)}^{(l)}\right) \mathrm{d} s-Z_{s}^{(l)} d B_{s}, & s \in[0, T], \\ Y_{s}^{(l)}=\xi_{s}^{(l)}, & s \in[T, T+D] .\end{cases}
$$

Let $\xi \in S^{2}(T, D+T), d_{1}^{(l)}$ and $d_{3}^{(l)}$ satisfy (D1) and (D2), and the function $g(\cdot)$ satisfies (A1) and (A2). If $d_{1}^{(1)}(v) \geq d_{1}^{(2)}(v), d_{3}^{(2)}(v) \geq d_{3}^{(1)}(v), 0 \leq v \leq T$,

then $\exists \lambda:=\lambda(C, L, T)>0$ s.t.

$$
\begin{gathered}
\left|Y_{t}^{(1)}-Y_{t}^{(2)}\right|^{2} \leq \lambda \int_{t}^{T}\left(d_{1}^{(1)}(v)-d_{1}^{(2)}(v)+d_{3}^{(2)}(v)-d_{3}^{(1)}(v)\right) \mathrm{d} v \\
\times E\left[\int_{T}^{T+D}\left|\xi_{r}\right|^{2} \mathrm{~d} r+\left|\xi_{T}\right|^{2}+\int_{t}^{T}|g(r, 0,0,0,0)|^{2} \mathrm{~d} r \mid \mathscr{F}_{t}\right] .
\end{gathered}
$$

Proof. Denote $y=Y^{(1)}-Y^{(2)}, z=Z^{(1)}-Z^{(2)}$, and then apply Itô's lemma for $\left|y_{s}\right|^{2} e^{\beta \dot{s}}$ :

$$
\begin{aligned}
\left|y_{u}\right|^{2}+E\left[\int_{u}^{T}\left(\beta\left|y_{v}\right|^{2}+\left|z_{v}\right|^{2}\right) e^{\beta(v-u)} \mathrm{d} s \mid \mathscr{F}_{u}\right] \\
=E\left[\int_{u}^{T} 2 y_{s} \mid g\left(v, Y_{v-d_{1}^{(1)}(v)}^{(1)}, Y_{v}^{(1)}, Z_{v}^{(1)}, Y_{v+d_{3}^{(1)}(v)}^{(1)}\right)\right. \\
\left.-g\left(v, Y_{v-d_{1}^{(2)}(v)}^{(2)}, Y_{v}^{(2)}, Z_{v}^{(2)}, Y_{v+d_{3}^{(2)}(v)}^{(2)}\right)\left|{ }^{2} e^{\beta(v-u)} \mathrm{d} v\right| \mathscr{F}_{u}\right],
\end{aligned}
$$


namely,

$$
\begin{aligned}
& \left|y_{t}\right|^{2}+E\left[\int_{t}^{T} \exp (\beta(s-t))\left(\left|z_{s}\right|^{2}+0.5 \beta\left|y_{s}\right|^{2}\right) \mathrm{d} s \mid \mathscr{F}_{t}\right] \\
& \leq 2 \beta^{-1} E\left[\int_{t}^{T} \mid g\left(s, Y_{s-d_{1}^{(1)}(s)}^{(1)}, Y_{s}^{(1)}, Z_{s}^{(1)}, Y_{s+d_{3}^{(1)}(s)}^{(1)}\right)\right. \\
& \left.-g\left(s, Y_{s-d_{1}^{(2)}(s)}^{(2)}, Y_{s}^{(2)}, Z_{s}^{(2)}, Y_{s+d_{3}^{(2)}(s)}^{(2)}\right)\left|2 e^{\beta(s-t)} \mathrm{d} s\right| \mathscr{F}_{t}\right] \\
& \leq \frac{8 C^{2}}{\beta} E\left[\int _ { t } ^ { T } \left(\left|Y_{s-d_{1}^{(1)}(s)}^{(1)}-Y_{s-d_{1}^{(2)}(s)}^{(2)}\right|^{2}+\left|y_{s}\right|^{2}+\left|z_{s}\right|^{2}\right.\right. \\
& \left.\left.+E\left[\left|Y_{s+d_{3}^{(1)}(s)}^{(1)}-Y_{s+d_{3}^{(2)}(s)}^{(2)}\right|^{2} \mid \mathscr{F}_{s}\right]\right) e^{\beta(s-t)} \mathrm{d} s \mid \mathscr{F}_{t}\right] \\
& \leq \frac{8 C^{2}}{\beta} E\left[\int _ { t } ^ { T } \left(2\left|y_{s-d_{1}^{(1)}(s)}\right|^{2}+2\left|Y_{s-d_{1}^{(1)}(s)}^{(2)}-Y_{s-d_{1}^{(2)}(s)}^{(2)}\right|^{2}+\left|y_{s}\right|^{2}+\left|z_{s}\right|^{2}\right.\right. \\
& \left.\left.+2 E\left[\left|y_{s+d_{3}^{(1)}(s)}\right|^{2} \mid \mathscr{F}_{s}\right]+2 \widehat{\mathbb{E}}\left[\left|Y_{s+d_{3}^{(1)}(s)}^{(2)}-Y_{s+d_{3}^{(2)}(s)}^{(2)}\right|^{2} \mid \mathscr{F}_{s}\right]\right) e^{\beta(s-t)} \mathrm{d} s \mid \mathscr{F}_{t}\right] \\
& \leq \frac{8 C^{2}+24 C^{2} L}{\beta} E\left[\int_{t}^{T} \exp (\beta(s-t))\left|y_{s}\right|^{2} \mathrm{~d} s \mid \mathscr{F}_{t}\right]+\frac{8 C^{2}}{\beta} E\left[\int_{t}^{T} \exp (\beta(s-t))\left|z_{s}\right|^{2} \mathrm{~d} s \mid \mathscr{F}_{t}\right] \\
& +\frac{16 C^{2}}{\beta} E\left[\int_{t}^{T} E\left[\left|\int_{s+d_{3}^{(1)}(s)}^{s+d_{3}^{(2)}(s)} g\left(r, Y_{r-d_{1}(r)}^{(2)}, Y_{r}^{(2)}, Z_{r}^{(2)}, Y_{r+d_{3}(r)}^{(2)}\right) \mathrm{d} r\right|^{2} \mid \mathscr{F}_{s}\right] e^{\beta(s-t)} \mathrm{d} s \mid \mathscr{F}_{t}\right] \\
& +\frac{16 C^{2}}{\beta} E\left[\int_{t}^{T}\left|\int_{s-d_{1}^{(1)}(s)}^{s-d_{1}^{(2)}(s)} g\left(r, Y_{r-d_{1}(r)}^{(2)}, Y_{r}^{(2)}, Z_{r}^{(2)}, Y_{r+d_{3}(r)}^{(2)}\right) \mathrm{d} r\right|^{2} e^{\beta(s-t)} \mathrm{d} s \mid \mathscr{F}_{t}\right] \text {. }
\end{aligned}
$$

We set $\beta=8 C^{2}$; then,

$$
\begin{aligned}
\left|y_{t}\right|^{2} \leq & (1+3 L) e^{\beta(T-t)} E\left[\int_{t}^{T}\left|y_{s}\right|^{2} \mathrm{~d} s \mid \mathscr{F}_{t}\right] \\
& +2 E\left[\int_{t}^{T} E\left[\left|\int_{s+d_{3}^{(1)}(s)}^{s+d_{3}^{(2)}(s)} g\left(r, Y_{r-d_{1}(r)}^{(2)}, Y_{r}^{(2)}, Z_{r}^{(2)}, Y_{r+d_{3}(r)}^{(2)}\right) \mathrm{d} r\right|^{2} \mid \mathscr{F}_{s}\right] e^{\beta(s-t)} \mathrm{d} s \mid \mathscr{F}_{t}\right] \\
& +2 E\left[\int_{t}^{T}\left|\int_{s-d_{1}^{(1)}(s)}^{s-d_{1}^{(2)}(s)} g\left(r, Y_{r-d_{1}(r)}^{(2)}, Y_{r}^{(2)}, Z_{r}^{(2)}, Y_{r+d_{3}(r)}^{(2)}\right) \mathrm{d} r\right|^{2} e^{\beta(s-t)} \mathrm{d} s \mid \mathscr{F}_{t}\right] \\
\leq & (1+3 L) e^{\beta(T-t)} E\left[\int_{t}^{T}\left|y_{s}\right|^{2} \mathrm{~d} s \mid \mathscr{F}_{t}\right] \\
& +2 E\left[\int_{t}^{T}\left(d_{3}^{(2)}(s)-d_{3}^{(1)}(s)\right) E\left[\int_{s+d_{3}^{(1)}(s)}^{s+d_{3}^{(2)}(s)}\left|g\left(r, Y_{r-d_{1}(r)}^{(2)}, Y_{r}^{(2)}, Z_{r}^{(2)}, Y_{r+d_{3}(r)}^{(2)}\right)\right|^{2} \mathrm{~d} r \mid \mathscr{F}_{s}\right] e^{\beta(s-t)} \mathrm{d} s \mid \mathscr{F}_{t}\right] \\
& +2 E\left[\int_{t}^{T}\left(d_{1}^{(1)}(s)-d_{1}^{(2)}(s)\right) \int_{s-d_{1}^{(1)}(s)}^{s-d_{1}^{(2)}(s)}\left|g\left(r, Y_{r-d_{1}(r)}^{(2)}, Y_{r}^{(2)}, Z_{r}^{(2)}, Y_{r+d_{3}(r)}^{(2)}\right)\right|^{2} \mathrm{~d} r e^{\beta(s-t)} \mathrm{d} s \mid \mathscr{F}_{t}\right] \\
\leq & e^{\beta(T-t)}(1+3 L) E\left[\int_{t}^{T}\left|y_{s}\right|^{2} \mathrm{~d} s \mid \mathscr{F}_{t}\right]+8 \int_{t}^{T}\left(\left(d_{1}^{(1)}(s)-d_{1}^{(2)}(s)\right)+\left(d_{3}^{(2)}(s)-d_{3}^{(1)}(s)\right)\right) e^{\beta(s-t)} \mathrm{d} s \\
& \cdot E\left[\int_{t}^{T}\left(C^{2}\left|Y_{r-d_{1}(r)}^{(2)}\right|^{2}+C^{2}\left|Y_{r}^{(2)}\right|^{2}+C^{2}\left|Z_{r}^{(2)}\right|^{2}+\left.C^{2}\left|Y_{r+d_{3}(r)}^{(2)}+\right| g(r, 0,0,0,0)\right|^{2}\right) \mathrm{d} r \mid \mathscr{F}_{t}\right] \\
\leq & (1+3 L) e^{\beta(T-t)} E\left[\int_{t}^{T}\left|y_{s}\right|^{2} \mathrm{~d} s \mid \mathscr{F}_{t}\right]+8 \int_{t}^{T} e^{\beta(s-t)}\left(\left(d_{1}^{(1)}(s)-d_{1}^{(2)}(s)\right)+\left(d_{3}^{(2)}(s)-d_{3}^{(1)}(s)\right)\right) \mathrm{d} s \\
& \cdot E\left[\int_{t}^{T}\left(C^{2}(2 L+1)\left|Y_{r}^{(2)}\right|^{2}+C^{2}\left|Z_{r}^{(2)}\right|^{2}+|g(r, 0,0,0,0)|^{2}\right) \mathrm{d} r+\int_{T}^{T+D}\left|\xi_{r}\right|^{2} \mathrm{~d} r \mid \mathscr{F}_{t}\right] .
\end{aligned}
$$


Therefore, there exists $\lambda^{\prime}:=\lambda^{\prime}(C, L, K)>0$ s.t.

$$
\begin{aligned}
\left|y_{t}\right|^{2} \leq & \lambda^{\prime} E\left[\int_{t}^{T}\left|y_{s}\right|^{2} \mathrm{~d} s \mid \mathscr{F}_{t}\right]+\lambda^{\prime} \int_{t}^{T}\left(\left(d_{1}^{(1)}(s)-d_{1}^{(2)}(s)\right)\right. \\
& \left.+\left(d_{3}^{(2)}(s)-d_{3}^{(1)}(s)\right)\right) \mathrm{d} s \\
& \times E\left[\left|\xi_{T}\right|^{2}+\int_{T}^{D+T}\left|\xi_{r}\right|^{2} \mathrm{~d} r+\int_{t}^{T}|g(r, \mathbf{0})|^{2} \mathrm{~d} r \mid \mathscr{F}_{t}\right] .
\end{aligned}
$$

Thus, from Gronwall inequality,

$$
\begin{aligned}
\left|y_{t}\right|^{2} \leq & \lambda \int_{t}^{T}\left(d_{1}^{(1)}(s)-d_{1}^{(2)}(s)+d_{3}^{(2)}(s)-d_{3}^{(1)}(s)\right) \mathrm{d} s \\
& \times E\left[\left|\xi_{T}\right|^{2}+\int_{t}^{T}|g(r, 0,0,0,0)|^{2} \mathrm{~d} r+\int_{T}^{T+D}\left|\xi_{r}\right|^{2} \mathrm{~d} r \mid \mathscr{F}_{t}\right] .
\end{aligned}
$$

Remark 1. If $g\left(v, Y_{v-d_{1}(v)}, Z_{v-d_{2}(v)}, Y_{v}, Z_{v}, \quad Y_{v+d_{3}(v)}\right.$, $\left.Z_{v+d_{4}(v)}\right)=f\left(v, Y_{v}, Z_{v}, Y_{v+d_{3}(v)}, Z_{v+d_{4}(v)}\right)$, then the delay and anticipated BSDE (1) is anticipated BSDEs in [15], and it is the result of Peng and Yang in [15].

Remark 2. In fact, the delay in (1) can go below $t=0$, namely, the DABSDE could have the following form:

$$
\begin{cases}Y_{v}=\theta_{v}^{\prime}, & v \in[-\tau, 0], \\ Z_{v}=\chi_{v}^{\prime}, & v \in[-\tau, 0], \\ -d Y_{v}=g\left(v, Y_{v-d_{1}(v)}, Z_{t-d_{2}(v)}, Y_{v}, Z_{v}, Y_{v+d_{3}(v)}, Z_{v+d_{4}(v)}\right) \mathrm{d} v-Z_{v} d B_{v}, & v \in[0, T], \\ Y_{v}=\theta_{v}, & v \in[T, D+T], \\ Z_{v}=\chi_{v}, & v \in[T, D+T],\end{cases}
$$

where $\tau>0, D>0$, and $d_{i}(\cdot), i=1, \ldots, 4$, are four positive continuous functions s.t.

(D3) $\exists D \geq 0$ s.t.

$$
\begin{aligned}
-\tau & \leq v-d_{1}(v) \leq v ;-\tau \leq v-d_{2}(v) \leq v ; v+d_{3}(v) \\
& \leq T+D ; v+d_{4}(v) \leq T+D, v \in[0, T] .
\end{aligned}
$$

(D4) $\exists K \geq 0$ s.t., for all nonnegative and integrable functions, $f(\cdot)$ satisfy

$$
\begin{aligned}
& \int_{t}^{T} f\left(u-d_{1}(u)\right) \mathrm{d} u \leq K \int_{-\tau}^{D+T} f(u) \mathrm{d} u ; \int_{t}^{T} f\left(u-d_{2}(u)\right) \mathrm{d} u \\
& \leq K \int_{-\tau}^{D+T} f(u) \mathrm{d} u ; \\
& \int_{t}^{T} f\left(u+d_{3}(u)\right) \mathrm{d} u \leq K \int_{t}^{D+T} f(u) \mathrm{d} u ; \\
& \int_{t}^{T} f\left(u+d_{4}(u)\right) \mathrm{d} u \leq K \int_{t}^{D+T} f(u) \mathrm{d} u,
\end{aligned}
$$

The existence and uniqueness of equation (39) can be obtained by the similar approach of proving Theorem 1 .

\section{Data Availability}

No data were used to support this study.

\section{Conflicts of Interest}

The authors declare that there are no conflicts of interest regarding the publication of this paper.

\section{Acknowledgments}

This research was based on NSF-China (nos. 11761028 and 11501009), Natural Science Foundation of Anhui Province (1508085JGD10), Yunnan Provincial Project (2018JS480), HTIT and RTFS-Yunnan Province, and Honghe University (nos. 2015HB061 and 2014HB0204).

\section{References}

[1] E. Pardoux and S. Peng, "Adapted solution of a backward stochastic differential equation," Systems Control Letters, vol. 14, pp. 61-74, 1990.

[2] Z. Chen and L. Epstein, "Ambiguity, risk, and asset returns in continuous time," Econometrica, vol. 70, no. 4, pp. 1403-1443, 2002.

[3] N. EI Karoui, S. Peng, and M. Quenez, "Backward stochastic differential equation in finance," Mathematical Finance, vol. 7, pp. 1-71, 1997.

[4] F. Coquet, Y. Hu, J. Mémin, and S. Peng, "Filtration-consistent nonlinear expectations and related g -expectations," Probability Theory and Related Fields, vol. 123, no. 1, pp. 1-27, 2002.

[5] L. Jiang, "Representation theorems for generators of backward stochastic differential equations and their applications," Stochastic Processes and Their Applications, vol. 115, no. 12, pp. 1883-1903, 2005. 
[6] L. Jiang, "Limit theorem and uniqueness theorem of backward stochastic differential equations," Science in China Series A: Mathematics, vol. 49, no. 10, pp. 1353-1362, 2006.

[7] Q. Lin, "Smallest g-supersolution with constraint," Applied Mathematics-A Journal of Chinese Universities, vol. 15, pp. 289-296, 2000.

[8] X. Mao, "Adapted solutions of backward stochastic differential equations with non-Lipschitz coefficients," Stochastic Processes and Their Applications, vol. 58, no. 2, pp. 281-292, 1995.

[9] M. Kobylanski, "Backward stochastic differential equations and partial equations with quadratic growth," The Annals of Probability, vol. 28, pp. 259-276, 2000.

[10] P. Briand, J. Lepetier, and J. San Martin, "One-dimensional backward stochastic differential equations whose coefficient is monotonic in \$y\$ and non-Lipschitz in \$z\$," Bernoulli, vol. 13, no. 1, pp. 80-91, 2007.

[11] P. Briand and Y. Hu, "Quadratic BSDEs with convex generators and unbounded terminal conditions," Probability Theory and Related Fields, vol. 141, no. 3-4, pp. 543-567, 2008.

[12] S. Fan and L. Jiang, "Finite and infinite time interval BSDEs with non-Lipschitz coefficients," Statistics \& Probability Letters, vol. 80, no. 11-12, pp. 962-968, 2010.

[13] Ł. Delong and P. Imkeller, "Backward stochastic differential equations with time delayed generators-results and counterexamples," The Annals of Applied Probability, vol. 20, no. 4, pp. 1512-1536, 2010.

[14] Ł. Delong and P. Imkeller, "On Malliavin's differentiability of BSDEs with time delayed generators driven by Brownian motions and Poisson random measures," Stochastic Processes and Their Applications, vol. 120, no. 9, pp. 1748-1775, 2010.

[15] S. Peng and Z. Yang, "Anticipated backward stochastic differential equations," The Annals of Probability, vol. 37, no. 3, pp. 877-902, 2009. 